\title{
Investigation of the association of Apgar score with maternal socio-economic and biological factors: an analysis of German perinatal statistics
}

\author{
Sebastian Straube $\cdot$ Manfred Voigt $\cdot$ Gerhard Jorch $\cdot$ \\ Ernst Hallier • Volker Briese · Ulrike Borchardt
}

Received: 29 January 2009 / Accepted: 17 August 2009 / Published online: 28 August 2009

(C) The Author(s) 2009. This article is published with open access at Springerlink.com

\begin{abstract}
Purpose To examine the relationship of 5-min Apgar score with maternal socio-economic and biological factors.

Methods We analyzed data from 465,964 singleton pregnancies (37-41 weeks' gestation) from the German perinatal statistics of 1998-2000. Using a logistic regression model we analyzed the incidence of low (0-6) 5-min Apgar scores in relation to these maternal factors: body mass index (BMI), age, previous live births, country of origin, occupation, single mother status, working during pregnancy, and smoking.

Results A low Apgar score was more common in overweight [adjusted odds ratio (OR) 1.24; $95 \%$ confidence interval (CI) 1.10-1.40; $P<0.001]$ and obese [OR 1.92 (95\% CI 1.67-2.20); $P<0.001]$ compared to normal weight women. A low Apgar score was also more common
\end{abstract}

S. Straube $(\bowtie) \cdot$ E. Hallier

Department of Occupational and Social Medicine,

University of Göttingen, Waldweg 37 B,

37073 Göttingen, Germany

e-mail: sebastian.straube@googlemail.com

M. Voigt

Institute for Perinatal Auxology,

Klinikum Südstadt, Rostock, Germany

G. Jorch

Department of Pediatrics, University of Magdeburg,

Magdeburg, Germany

V. Briese

Department of Obstetrics and Gynecology,

University of Rostock, Rostock, Germany

U. Borchardt

Research Institute for the Biology of Farm Animals (FBN),

Research Unit Genetics and Biometry, Dummerstorf, Germany for women aged $>35$ years compared to those aged 20 35 years [OR 1.35 (95\% CI 1.16-1.58); $P<0.001$ ]. Furthermore, odds of a low Apgar score were higher for women with no previous live births compared to those with one or more previous live births [OR 1.52 (95\% CI 1.37 $1.70) ; P<0.001]$. Socio-economic factors did not convincingly influence Apgar scores.

Conclusions There was an influence of the biological maternal factors age, BMI, and parity on the 5-min Apgar score. There was no convincing effect of socio-economic factors on Apgar score in our study population. Possible reasons for this are discussed.

Keywords Apgar score - Body mass index . Maternal age $\cdot$ Parity $\cdot$ Socio-economic factors

\section{Introduction}

The Apgar score [1] is commonly used to evaluate neonatal well-being immediately after birth. A total score of seven or more out of ten is considered an indication of a normal neonatal condition and a score of three or less is taken as a reason for especial concern. For its original use of predicting outcome in the neonatal period, the Apgar score is as useful today as it was when Virginia Apgar first described it. Taken individually, the 5-min Apgar score has been shown to be a better predictor of neonatal outcome than umbilical-artery blood $\mathrm{pH}$ [2], although it may be useful to combine these two predictors.

Even though the Apgar score was not originally intended to predict long-term health outcomes, it does nonetheless inform about prognosis beyond the neonatal period. It has been known for a while that the 5-min Apgar score is a good predictor of survival in infancy [3]. Likewise, it has 
been known for some time that a low Apgar score (0-3) for a prolonged period of time is predictive of subsequent disability [4]. A recent meta-analysis confirms that the outcome of neonates with a score of zero at $10 \mathrm{~min}$ is almost universally poor [5]. Interestingly, recent work showed that even transiently low Apgar scores are associated with lower IQ in later life [6].

Because the Apgar score is such an important indicator of subsequent outcome, knowing parameters associated with a low Apgar score is of clinical and epidemiological interest. Predicting low Apgar scores may allow the appropriate planning of neonatal care. Previous work showed that socio-economic as well as biological factors of the mother can be predictors of Apgar scores. For example, low social class, poor educational level and adverse social circumstances have been associated with lower Apgar scores in previous studies [7-9]. Of course adverse events during pregnancy and birth such as maternal infection can also result in a low Apgar score.

Our database constructed from data collected for German perinatal statistics provides a suitable means to investigate the influence of maternal biological and socio-economic factors on Apgar scores. The collection of perinatal statistics is mandatory in Germany. Data are compiled on a variety of maternal biological and socio-economic parameters throughout pregnancy. Data are also collected regarding the condition and well-being of the neonate following delivery, including Apgar scores. In this paper we aimed to analyze the relationship between 5-min Apgar scores and maternal biological and socio-economic parameters.

\section{Materials and methods}

We analyzed data of 465,964 singleton pregnancies and births from the perinatal statistics of eight German federal states: Bavaria, Brandenburg, Hamburg, Lower Saxony, Mecklenburg-Western Pomerania, Saxony, Saxony-Anhalt, and Thuringia. Data were collected between 1998 and 2000 and kindly passed on to us for analysis. We analyzed 5-min Apgar scores and their relation to a number of maternal biological and socio-economic factors: body mass index (BMI), age, previous live births, country of origin, occupation, whether the mother was a single parent or not, whether the mother worked during pregnancy or not, and number of cigarettes smoked per day (if any). To exclude length of gestation as a confounding factor, we restricted our analysis to cases with a duration of pregnancy of 37-41 completed weeks. This left 465,964 out of a total of 508,926 cases of singleton pregnancies in out database.

Nominal data are expressed as percent values. For bivariate analyses, the $\chi^{2}$ test was used. Multivariable logistic regression was used to assess the association between
Apgar scores and maternal biological as well as socio-economic factors. Odds ratios (OR) with regard to having a neonate with a 5-min Apgar score of 0-6 were calculated and adjusted for the following parameters:

- BMI Underweight (BMI < 18.5), overweight (BMI 2529.99 ), or obese (BMI $\geq 30$ ) women with reference to those of normal weight (BMI 18.5-24.99);

- Age Women aged $<20$ or $>35$ years with reference to those aged 20-35 years;

- Previous live births Women with no previous live births with reference to those with previous live births;

- Smoking status Smokers consuming 10 or less cigarettes a day and smokers consuming 11 or more cigarettes a day with reference to non-smokers;

- Maternal country of origin Women with a country of origin other than Germany with reference to women born in Germany (see Fig. 1);

- Maternal occupation Women in one of the first six occupational categories used in German perinatal statistics (Fig. 2) with reference to those in the most qualified occupational category (high level public employee, very highly skilled employee, etc.);

- Single mother status Women who described themselves as single mothers with reference to those who did not;

- Working during pregnancy Women who worked during pregnancy with reference to those who did not work.

OR were calculated with $95 \%$ confidence intervals (CI). A value of $P<0.05$ was considered statistically significant. All statistical analyses were performed with SPSS software, version 15.0.

\section{Results}

Table 1 illustrates that the distribution of 5-min Apgar scores is significantly influenced by maternal BMI, age, the presence or absence of previous live births, smoking status and number of cigarettes smoked per day, maternal occupation, maternal country of origin, being a single mother or not, and working during pregnancy or not. This means that these factors need to be considered in our logistic regression analysis (Table 2).

\section{Socio-economic factors}

Our regression analysis revealed that maternal occupation was not significantly associated with 5-min Apgar scores (Table 2; Fig. 1). Regarding maternal country of origin, only the comparison of the group of "other" countries (i.e., countries not otherwise classified in German perinatal statistics) with Germany yielded a statistically significant result [OR 1.73 (95\% CI 1.05-2.86)], see Table 2 and 
Fig. 1 Five-minute Apgar score according to country of origin of the mother. The terms for the geographical regions were translated as closely as possible from the German data collection form. "Central and Northern Europe" includes Austria, Switzerland, France, Belgium, the Netherlands, Luxembourg, Great Britain, Denmark, Sweden, Norway, and Finland. "Eastern Europe" includes the countries east of Germany

Fig. 2 Five-minute Apgar score according to maternal occupation
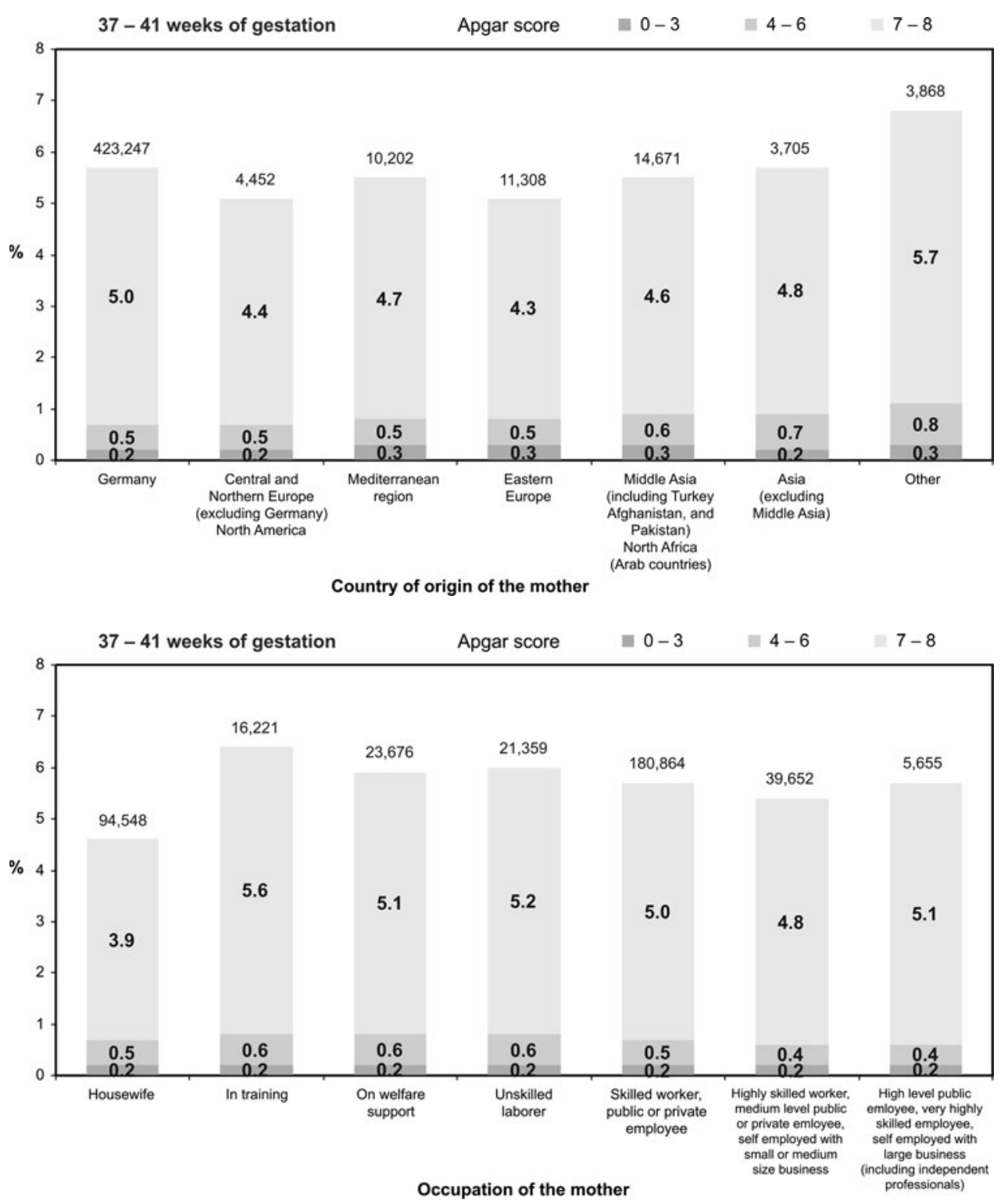

Fig. 2. Working during pregnancy or not and whether the mother was a single parent or not had no significant impact on the odds of having low Apgar scores. Similarly, for cigarette smoking our full model regression analysis did not reveal a significant association (Table 2). However, neonates of heavy smokers had significantly worse Apgar scores when a simpler regression model was used for analysis (not taking maternal country of origin and occupation into account, data not shown).

\section{Biological factors}

BMI was significantly associated with Apgar score. Both overweight [OR 1.24 (95\% CI 1.10-1.40); $P<0.001]$ and obese women [OR 1.92 (95\% CI 1.67-2.20); $P<0.001]$ had significantly higher odds of low Apgar scores (0-6) compared to normal weight women, as seen in Table 2.
Figure 3 shows the distribution of Apgar scores according to BMI. It is evident that lower Apgar scores are more common in the overweight and obese compared with the normal weight category. Maternal age and parity had significant influences on Apgar scores. Older women (above 35 years) had higher odds of low Apgar scores compared to women aged 20-35 years [OR 1.35 (95\% CI $1.16-1.58)$; $P<0.001]$. Absence of previous live births was also associated with higher odds of low Apgar scores [OR $1.52(95 \%$ CI 1.37-1.70); $P<0.001]$.

\section{Discussion}

Overall, we found no convincing associations between 5-min Apgar score and a variety of socio-economic factors of the mothers including country of origin, occupation, and smoking. 
Table 1 Distribution of Apgar scores in the population

\begin{tabular}{|c|c|c|c|}
\hline Parameter & Apgar $5 \min (0-6)$ & Apgar $5 \min (7-10)$ & $P\left(\chi^{2}\right.$ test $)$ \\
\hline \multicolumn{4}{|l|}{ Body mass index } \\
\hline$<18.50$ & 3.1 & 4.0 & \multirow[t]{4}{*}{$<0.001$} \\
\hline $18.50-24.99$ & 56.7 & 64.4 & \\
\hline $25.00-29.99$ & 24.1 & 21.5 & \\
\hline$\geq 30.00$ & 16.1 & 10.1 & \\
\hline \multicolumn{4}{|l|}{ Age (years) } \\
\hline$\leq 19$ & 4.4 & 3.5 & \multirow[t]{3}{*}{$<0.001$} \\
\hline $20-35$ & 82.4 & 86.7 & \\
\hline$\geq 36$ & 13.2 & 9.8 & \\
\hline \multicolumn{4}{|l|}{ Previous live births } \\
\hline 0 & 55.1 & 48.4 & \multirow[t]{2}{*}{$<0.001$} \\
\hline$\geq 1$ & 44.9 & 51.6 & \\
\hline \multicolumn{4}{|l|}{ Smoking } \\
\hline No & 80.4 & 83.9 & \multirow[t]{2}{*}{$<0.001$} \\
\hline Yes & 19.6 & 16.1 & \\
\hline \multicolumn{4}{|l|}{ Smokers } \\
\hline$\leq 10$ cigarettes per day & 69.2 & 73.1 & \multirow[t]{2}{*}{$<0.01$} \\
\hline$\geq 11$ cigarettes per day & 30.8 & 26.9 & \\
\hline \multicolumn{4}{|l|}{ Occupation of the mother } \\
\hline Housewife & 26.4 & 24.7 & \multirow[t]{6}{*}{$<0.001$} \\
\hline In training & 5.0 & 4.2 & \\
\hline On welfare support & 7.1 & 6.2 & \\
\hline Unskilled laborer & 6.3 & 5.6 & \\
\hline Skilled worker, public or private employee & 45.3 & 47.4 & \\
\hline $\begin{array}{l}\text { Highly skilled worker, medium level public } \\
\text { or private employee, self-employed } \\
\text { with small or medium size business }\end{array}$ & 8.5 & 10.4 & \\
\hline $\begin{array}{l}\text { High level public employee, very highly } \\
\text { skilled employed, self-employed with large } \\
\text { business (including independent professionals) }\end{array}$ & 1.4 & 1.5 & \\
\hline \multicolumn{4}{|l|}{ Country of origin of the mother } \\
\hline Germany & 87.3 & 89.8 & \multirow[t]{7}{*}{$<0.001$} \\
\hline $\begin{array}{l}\text { Central and Northern Europe (excluding Germany), } \\
\text { North America }\end{array}$ & 0.9 & 0.9 & \\
\hline Mediterranean region & 2.3 & 2.2 & \\
\hline Eastern Europe & 3.0 & 2.4 & \\
\hline $\begin{array}{l}\text { Middle Asia (including Turkey, Afghanistan, and Pakistan), } \\
\text { North Africa (Arab countries) }\end{array}$ & 3.9 & 3.1 & \\
\hline Asia (excluding Middle Asia) & 1.1 & 0.8 & \\
\hline Other & 1.4 & 0.8 & \\
\hline \multicolumn{4}{|l|}{ Single mother } \\
\hline Yes & 16.4 & 13.3 & $<0.001$ \\
\hline \multicolumn{4}{|l|}{ Worked during pregnancy } \\
\hline Yes & 43.7 & 48.9 & $<0.001$ \\
\hline
\end{tabular}

$n=465,964$ singleton pregnancies of 37-41 weeks' gestation. Frequencies of Apgar scores are given in percent

In our full regression model only the comparison of the heterogeneous group of "other" maternal countries of origin with Germany as a country of origin yielded a result that was just statistically significant. However, we did find a number of significant associations between biological parameters such as age, BMI and parity and 5-min Apgar scores.

Some limitations to our study need discussion. Our analysis relied on self-reporting of socio-economic parameters 
Table 2 Multivariate regression analysis

OR were calculated with regard to the odds of having a neonate with a 5-min Apgar score of 0-6. References were: Apgar score of 7-10, BMI of 18.5-24.99, age of $20-35$ years, $\geq 1$ previous live births, non-smoker, classified in the most qualified occupational category (high level public employee, very highly skilled employee, etc.), mother born in Germany, not being a single mother, and not working during pregnancy

* OR adjusted for the following parameters: BMI, age previous live births, smoking status, occupation of the mother, country of origin of the mother, single mother status, and working during pregnancy

${ }^{\text {a }} P<0.001$

Fig. 3 Five-minute Apgar score according to maternal BMI

\begin{tabular}{|c|c|}
\hline Parameter & OR $(95 \% \mathrm{CI})^{*}$ \\
\hline \multicolumn{2}{|l|}{ Body mass index } \\
\hline$<18.50$ & $0.82(0.62 ; 1.10)$ \\
\hline $25.00-29.99$ & $1.24(1.10 ; 1.40)^{\mathrm{a}}$ \\
\hline$\geq 30.00$ & $1.92(1.67 ; 2.20)^{\mathrm{a}}$ \\
\hline \multicolumn{2}{|l|}{ Age (years) } \\
\hline$\leq 19$ & $1.14(0.86 ; 1.51)$ \\
\hline$\geq 36$ & $1.35(1.16 ; 1.58)^{\mathrm{a}}$ \\
\hline \multicolumn{2}{|l|}{ Previous live births } \\
\hline None & $1.52(1.37 ; 1.70)^{\mathrm{a}}$ \\
\hline \multicolumn{2}{|l|}{ Smoking } \\
\hline$\leq 10$ cigarettes per day & $1.15(0.99 ; 1.34)$ \\
\hline$\geq 11$ cigarettes per day & $1.13(0.91 ; 1.41)$ \\
\hline \multicolumn{2}{|l|}{ Occupation of the mother } \\
\hline Housewife & $0.99(0.66 ; 1.48)$ \\
\hline In training & $0.96(0.62 ; 1.48)$ \\
\hline On welfare support & $0.96(0.64 ; 1.44)$ \\
\hline Unskilled laborer & $1.01(0.67 ; 1.53)$ \\
\hline Skilled worker, public or private employee & $0.88(0.61 ; 1.28)$ \\
\hline $\begin{array}{l}\text { Highly skilled worker, medium level public or private employee, } \\
\text { self-employed with small or medium size business }\end{array}$ & $0.85(0.57 ; 1.25)$ \\
\hline \multicolumn{2}{|l|}{ Country of origin of the mother } \\
\hline Central and Northern Europe (excluding Germany), North America & $0.99(0.56 ; 1.75)$ \\
\hline Mediterranean region & $1.06(0.74 ; 1.52)$ \\
\hline Eastern Europe & $1.18(0.82 ; 1.69)$ \\
\hline $\begin{array}{l}\text { Middle Asia (including Turkey, Afghanistan, and Pakistan), } \\
\text { North Africa (Arab countries) }\end{array}$ & $1.12(0.82 ; 1.54)$ \\
\hline Asia (excluding Middle Asia) & $1.31(0.72 ; 2.38)$ \\
\hline Other & $1.73(1.05 ; 2.86)$ \\
\hline \multicolumn{2}{|l|}{ Single mother } \\
\hline Yes & $1.06(0.91 ; 1.23)$ \\
\hline \multicolumn{2}{|l|}{ Worked during pregnancy } \\
\hline Yes & $1.03(0.92 ; 1.14)$ \\
\hline
\end{tabular}

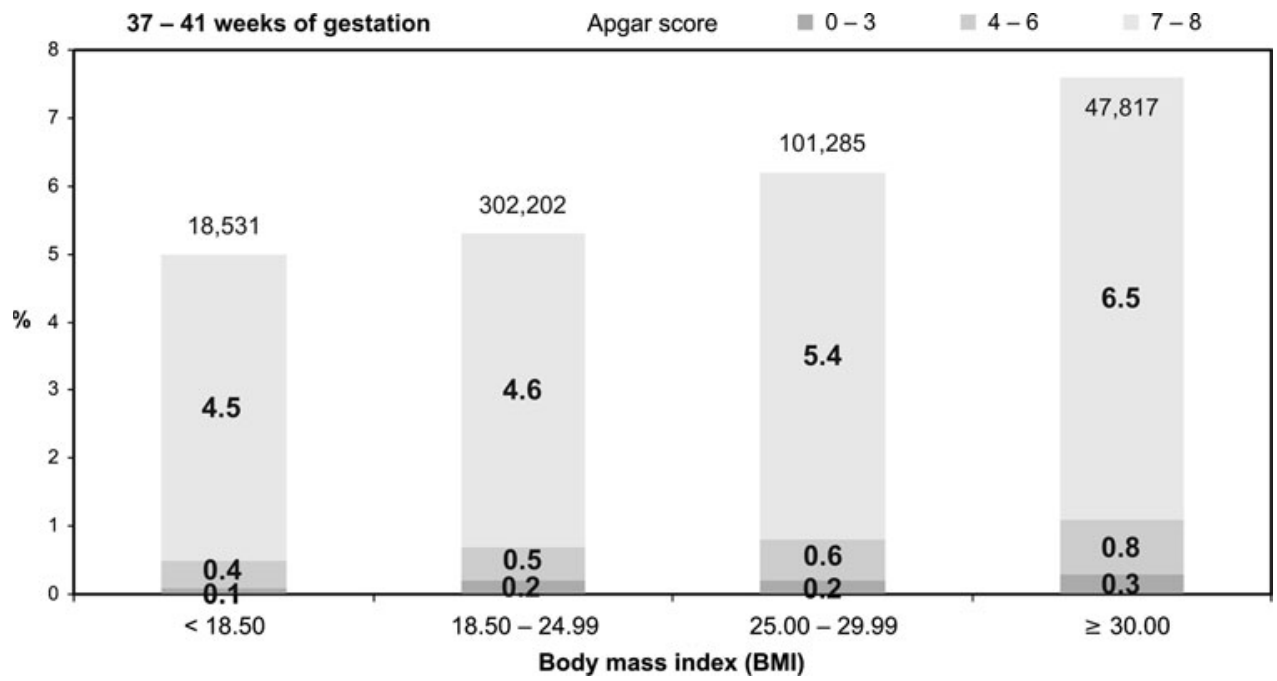


by the pregnant women. Self-reporting of socio-economic status and lifestyle habits (smoking) will not always be accurate. We also relied on the pre-existing classification of socio-economic parameters such as country of origin and occupation into rather broad categories. Some categories, for example, the German term "Mittlerer Osten" (Middle Asia) that was used in the classification system for maternal country of origin, are not precisely defined and thus may be open to interpretation. Likewise, the occupational categories are broad and accurate classification might be difficult in individual cases.

We are not alone in finding that associations of Apgar score with socio-economic parameters or lifestyle factors may be difficult to prove. For example, a recent study of a large cohort of over 50,000 children reported that the association between maternal smoking and Apgar score was eliminated after adjustment for confounders [10]. However, while we did not, with one exception, find significant associations of socio-economic factors with Apgar score in our full regression model, others did find such associations. A Finnish study of 2,912 women demonstrated a link between belonging to a lower social class, as evidenced by occupation and years of education, and having more infants with poor Apgar scores [7]. Another Finnish study confirmed the association between adverse social circumstances and low Apgar scores: children taken into custody and placed in foster care had lower Apgar scores compared with populationbased controls [8]. A Swedish study of 183,637 males born between 1973 and 1976 reported that mothers working in non-manual and self-employed occupations were less likely to have an infant with a low Apgar score than manual workers [9]. That study also found that the risk of a low Apgar score decreased with increasing maternal level of education.

The influence of socio-economic factors on Apgar scores seems to depend on the population studied and on precisely what socio-economic parameter is investigated. For example, a study from Spain found that perinatal complications, including an Apgar score of six or below, were not more frequent in the newborns of immigrant mothers compared to Spanish mothers [11]. An Australian study, however, reported that only $76.7 \%$ of babies born to indigenous Australian mothers fell into a "healthy baby" category, as characterized by being a live birth, a singleton, born after 37-41 completed weeks' gestation, having a birthweight of 2,500-4,499 g, and a 5-min Apgar score of at least 7 [12]. For non-indigenous mothers the rate of "healthy babies" was $85.0 \%$. A study from Washington State compared newborns of Somali immigrant women with those of black and white US-born women. Neonates born to Somali women were at increased risk of lower 5-min Apgar scores [13]. Given this dependence on study population, it is perhaps not surprising that we could not demonstrate a convincing influence of socioeconomic factors on Apgar scores even though we analyzed a large dataset. Our inability to find significant correlations may have to do with the above-mentioned limitations inherent in our data or it may be that socioeconomic differences and their impact on perinatal outcomes are less in Germany than in some other populations mentioned above. Another possible explanation is that our regression analysis, by taking confounding factors into account, eliminated some apparent influences of socioeconomic factors. Confounding and lifestyle factors may also provide an at least partial explanation of the results of the other studies. For example, in the study of indigenous Australian mothers more than half of these were smokers [12]. Alcohol consumption, although not mentioned in that study, may also have played a role.

Our result that working during pregnancy did not significantly increase the odds of having a newborn with a low Apgar score is encouraging. Employment during pregnancy does not seem to adversely affect neonatal outcome. This is in agreement with other work. Marbury and colleagues compared pregnancy outcomes of 7,155 women who worked between 1 and 9 months of pregnancy with outcomes of 4,018 women who were not employed. They found no differences in a range of parameters of neonatal health, including Apgar score [14].

A comparison of women aged 20-30 years with women over 40 from Taiwan $(n=400)$ found that the incidence of 5-min Apgar scores below 7 was significantly higher in the older group [15]. Similarly, we demonstrated increased odds of low Apgar scores for older mothers. A retrospective cohort study of nearly 3.9 million pregnancies and births from the USA found that infants born to teenage mothers of 17 or younger had a higher risk of low 5-min Apgar scores [16]. In contrast, we did not find a significant difference between mothers aged below 20 and those aged 20-35.

We also demonstrated an influence of maternal BMI on Apgar scores. This stands in contrast to some previous work. A population-based study of 60,167 deliveries from Wales found that a 5-min Apgar score below 7 was not significantly more common in the obese compared to normal weight women [17]. Likewise, a Danish study of 8,092 women found no differences between normal weight, overweight, and obese women with regard to Apgar score [18].

In conclusion, we demonstrated an effect of the biological parameters age, BMI, and parity on the 5-min Apgar score. We could not convincingly demonstrate an effect of socio-economic factors on Apgar score in our study population. 
Conflict of interest statement We declare that we have no conflicts of interest.

Open Access This article is distributed under the terms of the Creative Commons Attribution Noncommercial License which permits any noncommercial use, distribution, and reproduction in any medium, provided the original author(s) and source are credited.

\section{References}

1. Apgar V (1953) Proposal for new method of evaluation of newborn infant. Curr Res Anesth Analg 32:260-267

2. Casey BM, McIntire DD, Leveno KJ (2001) The continuing value of the Apgar score for the assessment of newborn infants. N Engl J Med 344:467-471

3. Drage JS, Kennedy C, Schwarz BK (1964) The Apgar score as an index of neonatal mortality: a report from the Collaborative Study of Cerebral Palsy. Obstet Gynecol 24:222-230

4. Nelson KB, Ellenberg JH (1981) Apgar scores as predictors of chronic neurologic disability. Pediatrics 68:36-44

5. Harrington DJ, Redman CW, Moulden M et al (2007) The longterm outcome in surviving infants with Apgar zero at 10 minutes: a systematic review of the literature and hospital-based cohort. Am J Obstet Gynecol 196:463 (e1-e5)

6. Odd DE, Rasmussen F, Gunnell D et al (2008) A cohort study of low Apgar scores and cognitive outcomes. Arch Dis Child Fetal Neonatal Ed 93:F115-F120

7. Hemminki E, Malin M, Rahkonen O (1990) Mother's social class and perinatal problems in a low-problem area. Int J Epidemiol 19:983-990
8. Kalland M, Sinkkonen J, Gissler M et al (2006) Maternal smoking behavior, background and neonatal health in Finnish children subsequently placed in foster care. Child Abuse Negl 30:1037-1047

9. Odd DE, Doyle P, Gunnell D et al (2008) Risk of low Apgar score and socioeconomic position: a study of Swedish male births. Acta Paediatr 97:1275-1280

10. Gilman SE, Gardener H, Buka SL (2008) Maternal smoking during pregnancy and children's cognitive and physical development: a causal risk factor? Am J Epidemiol 168:522-531

11. Pérez Cuadrado S, Muñoz Avalos N, Robledo Sánchez A et al (2004) Characteristics of immigrant women and their neonates. An Pediatr (Barc) 60:3-8

12. Graham S, Pulver LR, Wang YA et al (2007) The urban-remote divide for indigenous perinatal outcomes. Med J Aust 186:509512

13. Johnson EB, Reed SD, Hitti J et al (2005) Increased risk of adverse pregnancy outcome among Somali immigrants in Washington state. Am J Obstet Gynecol 193:475-482

14. Marbury MC, Linn S, Monson RR et al (1984) Work and pregnancy. J Occup Med 26:415-421

15. Jahromi BN, Husseini Z (2008) Pregnancy outcome at maternal age 40 and older. Taiwan J Obstet Gynecol. 47:318-321

16. Chen XK, Wen SW, Fleming N et al (2007) Teenage pregnancy and adverse birth outcomes: a large population based retrospective cohort study. Int J Epidemiol 36:368-373

17. Usha Kiran TS, Hemmadi S, Bethel J et al (2005) Outcome of pregnancy in a woman with an increased body mass index. BJOG 112:768-772

18. Rode L, Nilas L, Wøjdemann K et al (2005) Obesity-related complications in Danish single cephalic term pregnancies. Obstet Gynecol 105:537-542 